\title{
ensayo
}

\section{Comunicación para la salud en clave cultural y comunitaria}

\author{
Hernán Díaz \\ (Madrid, España) \\ Fundación de Educación para la Salud (FUNDADEPS) \\ Washington Uranga \\ (Buenos Aires, Argentina) \\ Universidad Nacional de La Plata
}

\section{Palabras clave \\ comunicación \\ salud \\ cultura \\ comunidad \\ relaciones \\ líderes \\ participación \\ democracia}

\begin{abstract}
Resumen
Vivimos en un mundo mediatizado, en el que existe una tendencia a pensar la comunicación en clave tecnológica e instrumental, poniendo la mirada en los medios masivos. Sin embargo, en particular en América Latina, los estudios de comunicación apuntan cada día con más insistencia al análisis de los procesos comunicacionales entendidos como la construcción de relaciones y el intercambio de sentidos entre los actores sociales que comparten un determinado contexto cultural. La comunicación para la salud ha sido entendida durante mucho tiempo como una herramienta para controlar y manipular las conductas individuales y colectivas. La revisión de esas experiencias y la puesta en práctica de una propuesta de comunicación que analiza los múltiples sentidos de las relaciones sociales, permite avanzar hacia la construcción de una perspectiva compleja de la realidad social. En esta perspectiva, lo comunicacional es una mirada que se articula con otras disciplinas y con los saberes vulgares, y los actores sociales tradicionalmente marginados o concebidos sólo como parte del objeto de estudio son artífices del cambio comunitario. Se propone que los individuos y grupos que forman una comunidad puedan tomar la palabra para plantear sus necesidades y problemas, y articularse para intervenir activamente en la búsqueda de soluciones, generando un cambio en los roles asignados tradicionalmente a los diferentes actores en materia de salud, en particular en lo referido a las relaciones de poder. La participación comunitaria es un derecho ciudadano y un imperativo democrático, pero es también una vía para aumentar la eficacia de las iniciativas sobre salud.
\end{abstract}




\title{
Health Communication in a Cultural- Community Framework
}

\begin{tabular}{c}
\hline Keywords \\
\hline communication \\
health \\
culture \\
community \\
relationships \\
leaders \\
participation \\
democracy
\end{tabular}

\begin{abstract}
We live in a world of media, in which there exists a tendency to think of communication in technological and instrumental terms, setting our sights on mass media. However, in the particular case of Latin America, communication studies point more and more towards analyses of communication processes which are understood as the creation of relationships and the exchange of senses between social actors who share a certain cultural context. Health communication has been understood for a long time as a tool for controlling and manipulating individual and collective conducts. Revising these experiences and carrying out a communication proposal which analyzes several aspects of social relationships, allows us to advance towards building a new and complex vision of social reality. According to this perspective, the communicative point of view is integrated with other disciplines and with common knowledge, and social actors who were traditionally outcasts or who were only considered as part of the object of study are now responsible for changes in their communities. It is intended that individuals and groups who form a community can speak out so as to express their needs and problems and coordinate themselves in order to be actively involved in the search for solutions, generating a change in the roles traditionally assigned to different actors in relation to health matters, in particular those regarding power relations. Community involvement is a civil right and a democratic imperative, but it is also a means for increasing the effectiveness of health initiatives.
\end{abstract}

\section{Cómo citar el artículo}

Díaz, H. y Uranga, W. (2011). Comunicación para la salud en clave cultural y comunitaria. Revista de Comunicación y Salud, 1(1), pp. 119-130.

DOI: http://doi.org/10.35669/revistadecomunicacionysalud.2011.1(1).119-130

\section{INTRODUCCIÓN}

La comunicación siempre ha estado presente en los procesos de salud. Sin embargo, el desarrollo de la comunicación como campo de análisis e intervención en las estrategias y programas de salud pública se vincula directamente con los cambios en la definición de salud que se produjeron a mediados del siglo XX. De los primeros planteamientos, que desde un modelo biomédico de recuperación del enfermo definieron a la salud simplemente como la "ausencia de enfermedad", se evolucionó hacia la definición actual que la concibe como "el completo estado de bienestar físico, mental y social, y no solo la ausencia de afecciones o enfermedades" (OMS, 1946: 1). Esta definición, fundamentada en una perspectiva bio-psico-social, trajo consigo una profunda transformación en las perspectivas y en las formas de abordar la salud 
pública, impulsada por las políticas de educación para la salud y promoción de la salud que tomaron fuerza en el último cuarto del siglo XX.

Pero tuvieron que pasar varias décadas desde aquella definición de salud, planteada en la Constitución de la Organización Mundial de la Salud en 1946, para que la perspectiva comunicacional se convirtiera en un componente de peso en los procesos comunitarios de salud. Es a partir de la $1^{\text {a }}$ Conferencia Mundial sobre Promoción de la Salud, celebrada en 1986 en la ciudad de Ottawa (Canadá), que la comunicación empieza a hacerse un lugar en los procesos de salud.

Las conclusiones de aquel cónclave se plasmaron en la Carta de Ottawa, un documento que definió la Promoción de la Salud como "el proceso de capacitar a las personas para que aumenten el control sobre los determinantes de la salud, para que la mejoren" (UIPES, 2003: 2). El concepto central en aquel planteamiento era el de control, o mejor, el autocontrol, entendido como dar las capacidades a las personas y colectivos para desarrollar conocimientos y habilidades que les permitan tomar decisiones autónomas y saludables.

La Carta de Ottawa señaló cinco estrategias principales para instalar de forma efectiva las políticas de promoción de la salud: la creación de una política pública saludable; la creación de entornos que apoyen la salud; el desarrollo de habilidades personales; la reorientación de los servicios sanitarios hacia la promoción de la salud; y el fortalecimiento de la acción comunitaria (OMS, 1986: 2-3). Todas estas estrategias indicaban, de una u otra manera, la necesidad de integrar a la comunicación como elemento transversal de los programas y acciones de educación para la salud y promoción de la salud.

La comunicación aparecía entonces como una herramienta fundamental en los procesos de salud, pero no todos tenían la misma idea de lo que implicaba integrar la comunicación en los proyectos y acciones de educación para la salud y promoción de la salud. En línea con las teorías hegemónicas por aquellos años, se instaló una concepción de la comunicación con un claro sentido instrumental, que la consideraba como una herramienta para manipular y controlar las conductas individuales y colectivas; una idea claramente vinculada con las primeras teorías de la comunicación, basada en perspectivas sistémicas y funcionalistas, que la concibieron como mera transmisión de información con fines persuasivos.

Estamos convencidos, porque los resultados de aquellas prácticas así lo demuestran, de que esa perspectiva de la comunicación impide un desarrollo adecuado de las estrategias de educación para la salud y promoción de la salud. En primer lugar, porque la comunicación no puede ser reducida a una cuestión de medios de comunicación, ni sus fines pueden ser únicamente la manipulación y la persuasión. En segundo lugar, porque está más que demostrado que cambiar conductas, promoviendo decisiones autónomas y saludables a nivel individual y colectivo, es un proceso extremadamente complejo en el que influyen múltiples actores y circunstancias de la vida de una comunidad.

Por ello, a lo largo de este artículo intentaremos abordar otra perspectiva de comunicación, una mirada que la concibe como el proceso de producción social de sentidos en el marco de un contexto social y cultural. Una perspectiva que intenta ofrecer elementos para abordar los 
proyectos comunitarios de salud desde una mirada compleja de la realidad, que contemple a las diferentes instancias de mediación de una comunidad, desde los medios de comunicación hasta los espacios y ámbitos de interrelación social; que incluya a los actores que tradicionalmente han sido hegemónicos en los procesos de salud, en general integrantes del ámbito sanitario, pero también a otros actores y líderes comunitarios con capacidad para influir en decisiones que afecten a la salud de la población; y que, por eso mismo, se convierta en una perspectiva integral e integradora, que ayude a consolidar y construir nuevas relaciones entre los actores, promoviendo procesos democráticos y participativos.

\section{LA COMUNICACIÓN, HABILIDAD PARA LA VIDA}

La comunicación, en sus más diferentes significados, constituye una característica fundamental de la vida contemporánea. Como dato constitutivo de la realidad, es un componente ineludible para comprender la dinámica de la vida social. Como disciplina y como categoría, tiene que ser considerada para entender el sentido de las prácticas históricas en las que estamos insertos.

En un mundo mediatizado como el que vivimos, existe una cierta tendencia a pensar la comunicación en clave tecnológica, poniendo la mirada en los múltiples medios masivos que forman parte de nuestras vidas y a los que estamos expuestos en nuestro día a día. Sin embargo, debemos recordar que, antes que nada, la comunicación es una característica esencialmente humana, que por eso mismo debe ser considerada dentro de las habilidades para la vida:

"Las habilidades para la vida son habilidades personales, interpersonales, cognitivas y físicas que permiten a las personas controlar y dirigir sus vidas, desarrollando la capacidad para vivir con su entorno y lograr que este cambie. Como ejemplos de habilidades individuales se pueden citar la toma de decisiones y la solución de problemas, el pensamiento creativo y crítico, el conocimiento de sí mismo y la empatía, las habilidades de comunicación y de relación interpersonal y la capacidad para hacer frente a las emociones y manejar el estrés. Las habilidades de vida tal y como se definen más arriba son elementos constitutivos fundamentales del desarrollo de habilidades personales para la promoción de la salud descritas como una de las áreas de acción clave en la Carta de Ottawa" (OMS, 1998).

La descripción que la Organización Mundial de la Salud (OMS) hace de las habilidades para la vida incluye de manera explícita y directa una serie de capacidades y destrezas reconocidas dentro del campo de la comunicación humana: "el conocimiento de sí mismo y la empatía, las habilidades de comunicación y de relación interpersonal y la capacidad para hacer frente a las emociones y manejar el estrés", retomando la cita que antecede. En esa cita no hay mención alguna, ni implícita ni explícita, a los medios de comunicación y al sistema que los contiene. Referirse a la comunicación, en primera y básica instancia, es hacer hincapié en la condición del varón y de la mujer como sujetos sociales capaces de generar una trama de relaciones de la que son parte, que los construye y que colaboran a construir. De esto estamos hablando cuando decimos comunicación en términos inicialmente humanos, de configuración de los sujetos sociales en el marco de un recorte territorial, geográfico o temático, dado. 


\subsection{LA COMUNICACIÓN EN CLAVE CULTURAL}

Cuando consideramos la relación entre comunicación y salud, nuestra mirada y nuestra preocupación -también nuestro "objeto de estudio"- se sitúa de manera primaria y primordial en el individuo situado en su contexto cultural más próximo, ámbito en el cual se relaciona con el mundo de los objetos y en el que ejerce su capacidad-potencialidad de transformación de su entorno físico y social. Este ámbito, así determinado, es el de la vida cotidiana. Y es esa mirada también la que nos permite referirnos a la comunicación, sobre todo y primordialmente, como un proceso que vincula o "intervincula" (White, 2007: 22) a los sujetos, comenzando por la relación de cada uno consigo mismo, tal como lo plantea Daniel Prieto Castillo (1999: 81).

A su vez, podemos decir que aquello que denominamos "vida cotidiana" es también el ámbito natural de lo que en las ciencias sociales nombramos como "prácticas sociales"; es decir, la resultante de la interacción entre los agentes sociales y el mundo de los objetos (las condiciones materiales que rodean a los agentes sociales). Hablamos de un tejido social que va cobrando forma en el marco de la cultura, entendida como un repertorio que nunca es homogéneo y que al mismo tiempo es dinámico y cambiante. Así, la cultura cobra densidad con aquellos significados que alcanzan durabilidad, que persisten en el tiempo y se concretan en comportamientos observables y que se interiorizan en forma de habitus, en el sentido que lo manifiesta Pierre Bourdieu (1985: 86-93).

Todo esto para sostener que la relación entre comunicación y salud sólo puede ser pensada y comprendida en el marco de las "habilidades humanas" mencionadas al comienzo. Es decir, más allá de toda mirada instrumental. Esto dicho a pesar de la "mediatización" de la sociedad en la que vivimos, donde los medios de comunicación construyen y constituyen ámbitos de definición de la cultura.

Sostenemos en consecuencia que la relación entre comunicación y salud sólo puede ser entendida desde una mirada que privilegia los vínculos entre los sujetos, individuos y actores sociales, y en la que las mediaciones, tal como las entiende Jesús Martín-Barbero (1987: 97), no son dispositivos sino ámbitos de transición que se convierten en espacios de resignificación permanente. Los individuos interpretan y comprenden los mensajes vinculados con la salud desde el espacio que generan las relaciones en la vida cotidiana, desde las marcas de la cultura que lo constituyen y desde el sistema de relaciones que, a modo de tejido social, los contiene.

Siguiendo a María Cristina Mata podemos sostener que la comunicación es el "conjunto de intercambios a partir de los cuales se van procesando identidades, normas, valores, se van articulando intereses, se van acumulando y legalizando saberes y poderes" y, por lo tanto, es un ámbito "privilegiado para la construcción del orden social", es decir, "como un terreno a partir de cual los diferentes actores propondrán sus propios sentidos de ese orden, que competirán entre sí para convertirse en hegemónicos" (Mata, 1988: 7).

Sostiene Eduardo Vizer que "toda forma de organización social instituye, y a su vez es instituida por estos procesos de construcción de sentido por medio de la comunicación (cultura), ya sea a través de conductas explícitas, rituales, ceremonias, gestos y estilos comunicativos, 
ya sean religiones, empresas, partidos políticos, etc. Se crean y recrean modelos culturales e identidades sociales reconocibles, se crean sistemas y códigos de diferenciación y reconocimiento social" (Vizer, 2006: 97). Así planteado, podemos afirmar también que las instituciones abocadas a la salud se constituyen en organizadoras de modelos culturales, que coronan formas de entender al sujeto, a su entorno y a su manera de relacionarse con su cuerpo, con la salud y con la enfermedad. De allí surgen prácticas que van configurando los modos de relacionamiento y de posicionamiento de los distintos actores que participan de los procesos de salud en una comunidad, en instituciones, programas o en las relaciones interpersonales.

Desde esta aproximación, la salud se entiende también como un fenómeno social, una realidad presente en la vida cotidiana de los sujetos o, visto desde otro lugar, en una práctica social atravesada por procesos comunicacionales. Por ese motivo, al afrontar problemáticas de salud en el marco de proyectos comunitarios, es necesario tomar en cuenta que toda acción en ese campo se ejerce sobre sujetos y grupos sociales que, además de dar significado a sus propios problemas y situaciones, aportan sobre todo significados subjetivos y sociales. La cuestión de la salud es, desde este punto de vista, ante todo una cuestión subjetiva y comunitaria, que requiere de un abordaje transversal nunca reducible a los mensajes mediáticos.

\subsection{UN CAMPO DE ACCIÓN, MÚLTIPLES SENTIDOS}

En América Latina se han ensayado, de manera consecutiva y simultánea y con éxito variado, diferentes estrategias de comunicación para la salud. Las prácticas de mercadeo aplicadas a las actividades sociales (marketing social); la educación por medio del entretenimiento (eduentretenimiento); el posicionamiento de temas en la agenda pública (abogacía de medios); alternativas de educación para la lectura crítica de los medios; los procesos de comunicación para la movilización social y las iniciativas de periodismo social o periodismo cívico son algunas de las estrategias implementadas en las últimas décadas. Todas estas estrategias han sido ensayadas, experimentadas y evaluadas. Pero la conclusión central del análisis de todos estos procesos, sigue siendo que la mirada tiene que ser puesta centralmente en los sujetos, hombres y mujeres, atravesados por las tensiones y las marcas de su propia historicidad.

Hemos señalado en la introducción de este artículo que en el campo de la comunicación para la salud conviven dos perspectivas de abordaje de la realidad. Por un lado, una perspectiva de marcado carácter instrumental para la cual la comunicación es mera transmisión de información desde un emisor a un receptor con el objetivo de manipular y controlar las conductas individuales y colectivas. Por otro lado, la perspectiva relacional de la comunicación, que la define como el proceso de producción social de sentidos en el marco de un contexto social y cultural, perspectiva que hemos venido planteando a lo largo de este artículo.

En el caso de la concepción instrumental, se concretó en prácticas en las que los comunicadores (o más reducido aún, los periodistas o publicistas) éramos llamados a aplicar nuestras técnicas una vez que los expertos en Epidemiología y Salud Pública ya habían definido hacia dónde querían torcer la realidad; prácticas que tenían el simple objetivo de difundir las actividades de determinado programa de salud entre la población a través de los medios masivos de comunicación durante la implementación de las acciones. Esta perspectiva, todavía vigente 
en las prácticas de muchos actores, se sustenta en la ilusión de que el emisor es capaz de controlar casi hipnóticamente las conductas de los receptores, lo que impide sacar provecho de las potencialidades que la comunicación ofrece como herramienta para facilitar e impulsar el cambio, de forma gradual e integrada, en proyectos más amplios que tomen en cuenta la complejidad social.

Como bien se encargó de señalar la Unión Internacional de Promoción y Educación para la Salud (UIPES), todas las evidencias señalan que influir en las conductas de los individuos y los grupos para que adopten conductas saludables es bastante complejo: "Si alguien deja de fumar, ¿lo hace porque ha visto un anuncio, ha hablado con un médico, sus amigos le han presionado, como resultado de iniciativas especiales, o ha tenido un pariente que ha muerto de cáncer de pulmón? El hecho es que es muy difícil saberlo. Sin embargo, lo importante es que esa persona ha dejado realmente de fumar" (UIPES, 2003: 2). Como se aprecia en esta definición, los individuos estamos mediados por múltiples influencias de nuestro entorno, no sólo por los medios masivos.

Esa evidencia ha hecho que en las últimas dos décadas haya cobrado fuerza y entidad una nueva forma de entender la comunicación para la salud, desde una concepción relacional. La comunicación se propone como una perspectiva integral e integradora, que intenta articular desde el comienzo los saberes y miradas de los diversos actores que participan en un proceso de salud. En esta perspectiva, las iniciativas de comunicación apuntan prioritariamente a conocer los lenguajes, el modo de relacionarse y los vínculos y mediaciones existentes en cada comunidad, para desde allí trabajar las problemáticas de salud sin escisión posible del espacio de lo cotidiano, de la cultura local, como ámbito ineludible de la representación de los problemas. Ya no se trata sólo de enviar información y difundir el mensaje que se quiere transmitir, sino de interactuar con los destinatarios, para construir sentidos colectivos y discursos que refieran a sus necesidades y problemas percibidos, que se discutan y resignifiquen en sus espacios y ámbitos colectivos.

Tomando en cuenta que toda práctica comunica, podemos decir al mismo tiempo que "la comunicación en salud busca constituirse en un proceso de carácter social que posibilite el reconocimiento, encuentro y diálogo de los diversos saberes, contribuyendo a consolidar la articulación entre los diferentes sectores sociales en torno a la salud" (Díez et al., 2004: 1).

Entendemos la cultura como un modo de organizar el movimiento permanente de la vida cotidiana (del ser y actuar cotidiano, sencillo y rutinario pero al mismo tiempo configurador de conductas, de modos de ser y actuar), como un principio organizador de la experiencia, mediante el cual los sujetos ordenan y estructuran sus acciones a partir del lugar que ocupan en las múltiples mediaciones que articulan y construyen sentido en una comunidad.

¿Hacia dónde encaminar entonces las iniciativas de comunicación para la salud? Indudablemente hacia estrategias que tengan como prioridad al sujeto en el marco de su comunidad. Esto supone también estrategias de comunicación que, antes que buscar el eventual cambio de conducta a través de técnicas basadas en el impacto publicitario, consoliden las posibilidades de que los sujetos lideren sus propios procesos. En ese sentido, las estrategias de comunicación se constituyen como un componente transversal que apunta a reforzar la autonomía y la capacidad de decisión de las personas antes que en un recurso para aumentar la eficacia de los mensajes. 
En definitiva, este cambio en la perspectiva comunicacional se centra no en la capacidad de controlar las conductas de los individuos desde una instancia superior (llámese, Estado o cualquier otra entidad promotora de una acción de salud), sino de que sean los propios individuos los que adquieran conocimientos y habilidades para adoptar decisiones más saludables. En resumen, pasamos de una idea de control a la de autocontrol, de la dependencia a la autonomía.

\subsection{COMUNICACIÓN PARA EL FORTALECIMIENTO COMUNITARIO}

La comunicación para la salud, entendida como "el uso y el estudio de las estrategias de comunicación para informar e influir sobre decisiones individuales y colectivas que mejoran la salud" (Instituto Nacional del Cáncer de Estados Unidos, 2004: 2-3), se presenta como una potente herramienta para facilitar y articular procesos de cambio comunitario relacionados con la salud. En ese planteo, la comunicación para la salud puede ayudarnos a conseguir una amplia variedad de objetivos:

- Incrementar el conocimiento de la población sobre un tema relacionado con la salud.

- Influir sobre las percepciones, creencias y actitudes, para reforzar o cambiar las normas sociales.

- Apoyar el aprendizaje de habilidades de salud.

- Mostrar los beneficios del cambio de conducta.

- Motivar para la acción.

- Incrementar la demanda y los recursos de los servicios de salud.

- Refutar mitos e ideas equivocadas.

- Abogar por una posición en un tema o política de salud.

- Fortalecer las relaciones entre organizaciones.

Como puede apreciarse, la gran mayoría de los objetivos de la comunicación para la salud refieren a los cambios a nivel individual y grupal respecto a determinadas conductas que favorecen o perjudican la salud. Sin embargo, los últimos dos elementos (abogar por una posición en un tema de salud y fortalecer las relaciones entre organizaciones) nos hablan precisamente de la potencialidad de la comunicación para construir de forma colectiva y participada nuevos escenarios y realidades.

Hemos señalado ya que fue a partir de la $1^{\text {a }}$ Conferencia Mundial de Promoción de la Salud que la comunicación ganó un lugar fundamental en los procesos de salud. Las cinco estrategias planteadas en la Carta de Ottawa para instalar de forma efectiva las políticas de promoción de la salud implicaban, directa o indirectamente, la necesidad de integrar la perspectiva comunicacional en los procesos de salud. Queremos en este punto poner nuestra mirada en una de esas cinco estrategias, el fortalecimiento de la acción comunitaria, porque a nuestro criterio tiene una trascendencia superior a la hora del cambio de modelo de salud y la aplicación efectiva de las políticas de promoción de la salud. 
Si la voluntad es fomentar la autonomía y el autocontrol de los individuos y los grupos en su salud, la participación comunitaria adquiere así un valor notable. Se trata sobre todas las cosas de que los diferentes individuos y grupos que forman una comunidad puedan tomar la palabra para plantear sus necesidades y problemas, y al mismo tiempo articularse para intervenir activamente en la búsqueda de soluciones. Esto supone un cambio en los roles asignados tradicionalmente a los diferentes actores en materia de salud, en particular en lo referido a las relaciones de poder. Como señaló la Organización Panamericana de la Salud:

"La participación social tiene que ver con las relaciones sociales, con la producción y usufructo de la cultura y, por ello, con las relaciones de poder. Por esto, estudiar y promover la participación social parte de asumir que hay quienes no participan o quienes no lo hacen de manera suficiente. Esto justifica impulsar procesos de participación social en la lógica del desarrollo de la salud comprendida en relación con una visión de bienestar definido por la propia sociedad" (OPS, 1992: 5).

Esta voluntad de fortalecer la participación de la comunidad conlleva también "un redimensionamiento de la relación entre saberes científicos y saberes populares" (Díaz, 2000: 70). Tradicionalmente, los proyectos comunitarios de salud se desarrollaban con la participación casi exclusiva de profesionales de distintas disciplinas científicas, en la mayoría de los casos de las ciencias de la salud, invalidando o menospreciando buena parte de los saberes vulgares o populares, saberes que los actores sociales utilizan como parte del sentido común en su vida cotidiana. Este es un aspecto que, en términos de comunicación y construcción de nuevas relaciones entre actores, cobra vital importancia. Estamos entonces ante "la necesidad de construir perspectivas transdisciplinares, visiones que trasciendan el ámbito mismo de las disciplinas científicas para incorporar a la comunidad desde el origen de los procesos de cambio. Una perspectiva en la que los actores sociales participen activamente en el reconocimiento de su historia y su presente para proyectar su futuro" (Díaz, 2000: 80).

La incorporación de "nuevos" actores en los procesos de salud para que desempeñen un papel activo, con capacidad para liderar y decidir el rumbo a adoptar, es, primero que todo, un derecho de ciudadanía y un imperativo democrático. Pero, además, es una vía para aumentar la eficacia y la eficiencia de las iniciativas sobre salud, porque esos actores (líderes comunitarios, referentes, colectivos ciudadanos, plataformas de organizaciones civiles...) son los interlocutores legítimos de la comunidad, conocen mejor a los destinatarios de la acción, saben mejor cómo relacionarse con ellos y cómo lograr su participación activa en las propuestas de cambio.

En definitiva, se trata de avanzar hacia la construcción de una perspectiva compleja de la realidad social, en la que lo comunicacional sea una mirada que se articule con otras disciplinas y con los saberes vulgares y en la que actores sociales tradicionalmente marginados o concebidos sólo como parte del objeto de estudio sean artífices del cambio comunitario. Es evidente que, desde la perspectiva propuesta, la comunicación para la salud puede facilitar e impulsar determinados procesos de cambio, pero tiene también sus dificultades y deben considerarse desde el principio.

En primer lugar, generar proyectos y acciones de promoción de la salud con voluntad de participación comunitaria debe tener en cuenta la complejidad que ello conlleva. Es esperable 
que aquellos actores que tradicionalmente han llevado la voz de mando en esos proyectos, generalmente del ámbito sanitario, muestren su oposición y pongan obstáculos ante la pérdida del control (y de la cuota de poder) que implica tener en cuenta las problemáticas, necesidades y formas de gestión planteadas por otros actores representativos de la comunidad. Poner en marcha estos procesos participativos supone dar un lugar preeminente a la comunicación, primero que todo en clave interna, para el reconocimiento mutuo de los actores que participan, la construcción de una nueva identidad colectiva y la búsqueda de consensos sobre los objetivos a seguir y la forma de llegar a ellos.

En segundo lugar, estos procesos participativos pueden enriquecer en gran medida los proyectos comunitarios de salud y aumentar su viabilidad y eficacia, pero también pueden demandar tiempos más largos en su diseño e implementación. Es bastante habitual que los diferentes actores propongan diferentes visiones de la realidad y propongan estrategias distintas para generar el cambio, y todo proceso de negociación y búsqueda de consensos requiere de tiempo. Antes que alarmarse por las demoras que se puedan producir en la gestión, hay que considerar que ese proceso es también parte del aprendizaje que los actores participantes obtienen en término de ejercicio de derechos de ciudadanía, que se añaden a los beneficios que obtendrán con los cambios saludables que se pretenden promover.

\section{CONCLUSIÓN}

Estamos convencidos de que la comunicación para la salud ofrece múltiples elementos para promover y facilitar cambios saludables en nuestras sociedades, tanto en lo que refiere al fortalecimiento de los actores y redes comunitarias como en la promoción de nuevas concepciones y habilidades individuales y colectivas relacionadas con la salud. Su integración en los programas y acciones de educación para la salud y promoción de la salud, desde el momento mismo de su gestación y diseño y a lo largo de todo el proceso, puede favorecer el desarrollo de esas iniciativas para conseguir un mayor impacto en la sociedad.

Pero, a la vez, debemos situar en su justa medida los alcances y limitaciones de la comunicación para la salud, porque su potencial de ninguna manera es suficiente para transformar mágicamente la realidad. La comunicación puede promover en la ciudadanía conductas saludables para prevenir enfermedades y mejorar su calidad de vida, pero no puede garantizar que los individuos adopten efectivamente esas conductas. Puede aportarnos herramientas para informar a la comunidad sobre cuándo demandar atención y cómo hacer un uso adecuado de los recursos del sistema sanitario, pero no puede cubrir la carencia de esos recursos. Puede facilitar y dinamizar cambios de conductas de salud pero difícilmente podrá hacer que sean sostenibles en el tiempo si no van acompañados por otras acciones que apoyen ese cambio (Instituto Nacional del Cáncer de Estados Unidos, 2004: 3).

Nuestra propuesta tiene su eje en la comunicación como hecho humano, relacional. Entendemos que la comunicación no puede ser entendida apenas como instrumentos o habilidades. En su mirada más compleja, la comunicación es una dimensión transversal de la vida de las personas y de la cultura que entreteje las relaciones sociales. En ese escenario todos los actores y todos los saberes pueden y deben ser puestos al servicio de la mejor calidad de vida. 
La comunicación para la salud, aún más allá de las diferentes estrategias que se pongan en juego, tiene que basarse en el reconocimiento de la trama cultural y de la importancia de todos los actores, con saberes diferenciados pero todos imprescindibles, útiles y valiosos para mejorar las condiciones de vida de la población. La promoción de conductas saludables no es el resultado de campañas o de acosos publicitarios, sino la consecuencia lógica de la apropiación de otro sentido respecto a la calidad de vida y a la salud por parte de la ciudadanía.

\section{Referencias}

Bourdieu, Pierre (1985). Dialogue a propòs de l'histoire culturelle. París, Acte de la Recherche en Sciencies Sociales, No. 59.

Díaz, Hernán Alfredo (2000): Procesos de comunicación para la salud: criterios y acciones para la prevención del infarto en un proyecto de cambio comunitario en Argentina. Tesina de Investigación del Magíster en Técnicas de Comunicación en Servicios Sociales. Facultad de Ciencias de la Información de la Universidad Complutense de Madrid.

Díez, Patricia; Ghitia, Adriana; Jait, Andrea y Oliva, Karina (2004). Buenos Aires Salud 2003, una experiencia innovadora de comunicación en salud. Disponible en www.catedras.fsoc.uba.ar/zucchelli/BuenosAiresSalud2003unaexperienciainnovadora.pdf. Recuperado el 14/10/2010.

Instituto Nacional del Cáncer de los Estados Unidos (2004): Making health communication programs work. Disponible en http://www.cancer.gov/pinkbook. Recuperado el 15/09/2010.

Martín-Barbero, Jesús (1987); De los medios a las mediaciones. Barcelona, G. Gili.

Mata, María Cristina (1988). Comunicación popular: de la exclusión a la presencia. Córdoba, Argentina. Mimeo.

Organización Mundial de la Salud (1946). Constitución de la Organización Mundial de la Salud. Disponible en http://apps.who.int/gb/bd/PDF/bd47/SP/constitucion-sp.pdf. Recuperado el 15 de septiembre de 2010.

Organización Mundial de la Salud (1986). Carta de Ottawa. Primera Conferencia Mundial de Promoción para la Salud. Disponible en www.paho.org/spanish/HPP/OttawaCharterSp.pdf. Recuperado el 15 de septiembre de 2010.

Organización Mundial de la Salud (1998). Promoción para la Salud. Glosario. Disponible en http://www.bvs.org.ar/pdf/glosario_sp.pdf. Recuperado el 5 de octubre de 2010.

Organización Panamericana de la Salud (1992): Desarrollo y fortalecimiento de los sistemas locales de salud en la transformación de los sistemas nacionales de salud. La participación social en el desarrollo de la salud. Documento de trabajo de la Organización Panamericana de la Salud (OPS).

Prieto Castillo, Daniel (1999). La comunicación en la educación. Buenos Aires, CICCUS - La Crujía. 
Unión Internacional de Promoción de la Salud y Educación para la Salud (2003): La evidencia de la eficacia de la Promoción de la Salud. Informe para la Comisión Europea elaborado por la Unión Internacional de Promoción de la Salud y Educación para la Salud. Parte Uno: Documento base. Versión española traducida, adaptada y editada por el Ministerio de Sanidad y Consumo del Gobierno de España.

Uranga, Washington (2007). Mirar desde la comunicación. Buenos Aires. Disponible en http://www.conabip.gov.ar/Contenidos/Documentos/04Textodeprofundizacion.pdf. Recuperado el 16 de octubre de 2010.

Vizer, Eduardo (2006. 2da. Edic.). La trama (in)visible de la vida social. Buenos Aires, La Crujía White, Robert (2007) Comunicar comunidad. Aportes para una ética de la comunicación pública. Buenos Aires, La Crujía. 